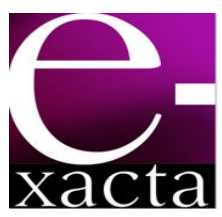

ISSN: 1984-3151

\title{
REMOÇÃO dO FENANTRENO POR BIOMASSA VIVA E INATIVADA DE CUNNINGHAMELLA ELEGANS UCP0542
}

\author{
REMOVAL OF PHENANTHRENE BY BIOMASS LIVE AND \\ INACTIVATED OF CUNNINGHAMELLA ELEGANS UCP0542
}

\author{
Marta Cristina Freitas Silva ${ }^{1,4}$, Adriana Almeida \\ Antunes $^{1,4}$, Clarissa Isabel Matos Lins ${ }^{2,4}$, Antonio \\ Helder Parente ${ }^{3}$, Sônia Valéria Pereira ${ }^{3}$, Galba \\ Maria Campos-Takaki ${ }^{4}$
}

1 Pós-Doutoranda em Biotecnologia - PNPD - CAPES, FACEPE. martacfs@yahoo.com.br.

2 Doutoranda de Engenharia Química - UNICAMP, Brasil. rissabel@gmail.com.

3 Laboratório de Tecnologia Ambiental - LABTAM, Instituto de Tecnologia de Pernambuco, Recife-PE, Brasil. helderparente@gmail.com. soniaitep@gmail.com.

4 Núcleo de Pesquisas em Ciências Ambientais, Centro de Ciências e Tecnologia, Universidade Católica de Pernambuco, Rua Nunes Machado no 42 Bloco J Boa Vista - CEP: 50050-590. co J Boa Vista - CEP: 50050-590. adri antunes@yahoo.com.br. takaki@unicap.br.

Recebido em: 21/02/2013 - Aprovado em: 20/04/2013 - Disponibilizado em: 11/06/2013

RESUMO: Realizou-se estudos avaliando a ação da biomassa viva e inativada de Cunninghamella elegans crescida, visando à remoção do hidrocarboneto aromático policíclico fenantreno. Monitorou-se o processo de remoção do fenantreno a cada $24 \mathrm{~h}$ por espectrofotometria, de acordo com um planejamento fatorial completo de $2^{2}$ e, como variáveis independentes 0,1 e 0,2 mM de fenantreno, na presença de 0,6, 3,3 e $6 \%$ de glicose e variável resposta o conteúdo de fenantreno removido. Os resultados obtidos indicaram que tanto a biomassa inativada como viva removeram, respectivamente, 90,0\% e 82,0\% a concentração de 0,2mM de fenantreno na presença da menor concentração de glicose (0,6\%). A biomassa inativada de $C$. elegans demonstrou ser um sorbente com elevado potencial biotecnológico para a remediação de áreas poluídas com hidrocarbonetos aromáticos policíclicos.

PALAVRAS-CHAVE: Fenantreno. Cunninghamella elegans. Biomassa. Remoção.

ABSTRACT: Studies were carried out evaluating the action of the life and inactivated biomass of Cunninghamella elegans grown to removal polycyclic aromatic hydrocarbon phenanthrene. The removal process was monitored each $24 \mathrm{~h}$ by spectrophotometry, de acordo com according to full factorial design $2^{2}$, and independent variables the phenanthrene concentrations $[0.1$ and $0.2 \mathrm{mM}]$, and the content of phenanthrene removed as variable response. The results showed that both inactivated and live biomass from C. elegans removed $85.0 \%$ and $90.0 \%$, respectively, of phenantrene of $0.2 \mathrm{mM}$ on glucose (0.6) concentration. The inactivated biomass from C. elegans shows a sorbent with high biotechnological potential for bioremediation of polluted area with polycyclic aromatic hydrocarbons.

KEYWORDS: Phenanthrene. Cunninghamella elegans. Biomass. Removal. 


\section{INTRODUÇÃO}

Os hidrocarbonetos policíclicos aromáticos (HAP's) são poluentes amplamente distribuídos no meio ambiente quando resultam da queima de combustíveis fósseis e de atividades industriais (CERNIGLIA; GIBSON, 1979; DEZIE'L et al., 1996; CERNIGLIA; VAN BAALEN; GIBSON, 1980; CERNIGLIA, 1992; 1997; CERNIGLIA; SUTHERLAND, 2001). Esses estão presentes como poluentes orgânicos, com propriedades cancerígenas e tóxicas (SAKAY et al., 1985; MALISZEWSKA-KORDYBACH; SMRECZAK, 2003; ZHANG, WANG; XIE, 2011). Estima-se que mais de $90 \%$ da carga total de HAP esteja nos solos superficiais (WILD; JONES, 1995).

A degradação microbiana de HAP tem recebido uma atenção especial como uma possível estratégia para a biorremediação de HAP's em solos contaminados (JOHNSEN; WICK; HARMS, 2005). Grandes quantidades de compostos orgânicos e inorgânicos são liberadas para o meio ambiente a cada ano como resultado de atividades humanas (VIÑAS et al., 2005).

Uma estratégia para descontaminação dos HAP's do ambiente é através da biorremediação, que é a utilização do processo ou atividade biológica para transformar os contaminantes em substâncias inertes (HOLLIGER; WOHLFAHRT; DIEKERT, 1998; BOUCHEZ et al., 2000; SAMANTHA et al., 2002; WU; WANG; XING, 2005).

O alto custo para promover a remoção de HAP's do ambiente induziu ao grande interesse para a busca de micro-organismos com potencial para os processos de biorremediação e detoxificação de locais poluídos (SUTHERLAND, 1992; BOONCHAN; BRITZ; STANLEY, 2000; WU; WANG; XING, 2005; LEITÃO, 2009).

A biorremediação de sítios contaminados depende do potencial e habilidade microbiana na

e-xacta, Belo Horizonte, v. 6, n. 1, p. 1-8. (2013). Editora UniBH. Disponível em: www.unibh.br/revistas/exacta/ transformação de poluentes, essencialmente em compostos inofensivos ou, no mínimo, menos perigosos (HOFRICHTER; BUBLITZ; FRITSCHE, 1995; EL FANTROUSSI; AGATHOS, 2005; PAZARLIOGLU; TELEFONCU, 2005, TAO et al., 2009). O fungo $C$. elegans metaboliza HAP's e, por isso, é importante para processos de biorremediação (BOONCHAN; BRITZ; STANLEY, 1998; BANFOURTH; SINGLETON, 2005; GENTILI et al., 2006). C. elegans metaboliza inicialmente HAP's em trans-hidrodióis, fenóis, quinonas e epóxidos de hidrodióis. C. elegans oxida fenantreno nas posições 1,2 e 3,4 para formar trans-dihidrodióis. As enzimas agem nas posições 9 e10, que são os principais locais para a oxidação de fenantreno (CERNIGLIA, 1979; BRODKORB; LEGGE, 1992; BARCLAY; FARQUHAR; LEGGE, 1995; LEITÃO, 2009).

Essa biotecnologia vem sendo utilizada há vários anos em outros países e, em certos casos, apresenta menor custo e maior eficiência na remoção dos contaminantes do que as técnicas físicas e químicas (como incineração e lavagem do solo), sendo atualmente utilizada em escala comercial no tratamento de diversos resíduos e na remediação de áreas contaminadas (BAMFORTH; SINGLETON, 2005; ZHU; AITKEN, 2010).

Por outro lado, a degradação dos HAP's no solo pode ser limitada pela incapacidade dos microorganismos autóctones em metabolizar eficientemente esses compostos, devido à falta de nutrientes ou pela baixa disponibilidade dos HAP's aos micro-organismos degradadores (JOHNSEN; WICK; HARMS, 2005). Neste trabalho foi investigada a habilidade da biomassa viva e inativada de $C$. elegansnaremoçãode fenantreno, de acordo com um planejamento fatorial completo de $2^{2}$, tendo como variáveis independentes as concentrações 0,1 e 0,2 mM na presença de 0,6, 
3,3 e $6 \%$ de glicose e, como variável resposta, o conteúdo removido de fenantreno.

\section{Material e Métodos}

\subsection{MATERIAIS}

O micro-organismo Cunninghamella elegans (UCP 542) foi isolado do sedimento de mangue do Município de Rio Formoso, Pernambuco, Brasil, catalogado e depositado na Coleção do Núcleo de Pesquisa em Ciências Ambientais - NPCIAMBUniversidade Católica de Pernambuco - UNICAP (GOMES et al., 2000). A coleção é registrada no World Federation Culture Collection-WFCC. A linhagem foi mantida em meio de cultivo Batata Dextrose Agar (BDA) a $5^{\circ} \mathrm{C}$.

Fenantreno: o fenantreno foi adquirido da AldrichSigma, United Kingdom. A solução estoque foi preparada dissolvendo-se o fenantreno a $10 \mathrm{mM}$ em acetona, filtrada em membrana Millipore de $0,45 \mu \mathrm{m}$ e armazenada em frasco âmbar, à temperatura de $5^{\circ} \mathrm{C}$.

\subsection{MÉTODOS}

Produção de biomassa: Os esporangíolos de $C$. elegans cresceram durante sete dias a $28^{\circ} \mathrm{C}$ em placas de Petri contendo o meio Yeast Malt Agar (YMA). Utilizando-se um cultivo monospórico de $C$. elegans, foi realizada a padronização de inóculo através da contagem de esporangíolos em hematocitômetro para uma concentração final de $10^{7}$ esporangíolos $/ \mathrm{mL}$. Cerca de $5 \%$ do inóculo foi transferido para de Erlenmeyers de $1000 \mathrm{~mL}$ de capacidade, contendo $600 \mathrm{~mL}$ do meio de cultura, como descrito por Hesseltine e Anderson (1957). Em seguida, os frascos foram incubados a $28^{\circ} \mathrm{C}$, por 96 horas, sob agitação orbital de $150 \mathrm{rpm}$. Ao final do crescimento, a biomassa foi coletada por filtração em membrana de fibra sintética de silkscreen $(0,8 \mu \mathrm{m})$, seguida de duas lavagens com água deionizada gelada e mantida à temperatura de $5^{\circ} \mathrm{C}$.

Inativação da Biomassa: Cerca de $5 \mathrm{~g}$ de micélio fresco de $C$. elegans foram imersos em $20 \mathrm{~mL}$ de água deionizada e inativados em autoclave por 15 minutos, à temperatura de $121^{\circ} \mathrm{C}$. O micélio inativado foi separado por filtração através de um filtro de nylon serigrafia $(0,8 \mu \mathrm{m})$ (MORAES FILHO et al., 2009).

Cinética de remoção do fenantreno pela biomassa de C. elegans: A remoção do fenantreno foi realizada em frascos de Erlenmeyer de $500 \mathrm{~mL}$ de capacidade contendo $100 \mathrm{~mL}$ das soluções de fenantreno 0,1 e $0,2 \mathrm{mM}$ e adicionadas as concentrações 0,6 e $6,0 \%$ de glicose, baseadas respectivamente, nos trabalhos de (CAMPOSTAKAKI; DIETRICH; BEAKES, 1983) e (ANDRADE et al., 2000). A concentração 3,3\% foi de acordo com o ponto central do planejamento fatorial completo de $2^{2}$. Em seguida nos frascos foram adicionados, de forma estéril, $5 \mathrm{~g}$ das biomassas viva e/ou inativada, mantidos sob agitação orbital de $150 \mathrm{~Hz}$ à temperatura de $28^{\circ} \mathrm{C}$. A cinética de remoção do fenantreno foi realizada através da coleta de alíquotas de 2,00 mL, a cada 24 horas até que se completasse o período de 96 horas.

Determinação do teor de fenantreno: $O$ teor de fenantreno foi determinado por espectrofotometria, através de uma curva de calibração com uma solução de fenantreno de 1-10 mM, no comprimento de onda de $250 \mathrm{~nm}$, segundo Veintemilia (2006).

Os cálculos de todos os testes foram realizados utilizando o programa STATISTIC versão 6.0 da Statsoft Inc., USA. 


\section{Resultados e Discussão}

\subsection{EFEITOS DE DIFERENTES} CONCENTRAÇÕES DE GLICOSE NA REMOÇÃO

\section{DE FENANTRENO}

Estudos realizados com as biomassas viva e inativadade $C$. elegans na remoção de fenantreno $(0,1$ e $0,2 \mathrm{mM})$ na presença de diferentes concentrações de glicose estão apresentados na Figura 1 ( $A$ e $B$ ) e na Figura 2 ( $A$ e $B$ ).

A Figura 1 apresenta a biomassa inativada de $C$. elegans em diferentes concentrações de glicose na presença de fenantreno 0,1 mM (Figura 1-A); e em diferentes concentrações de glicose na presença de fenantreno 0,2mM (Figura 1-B).
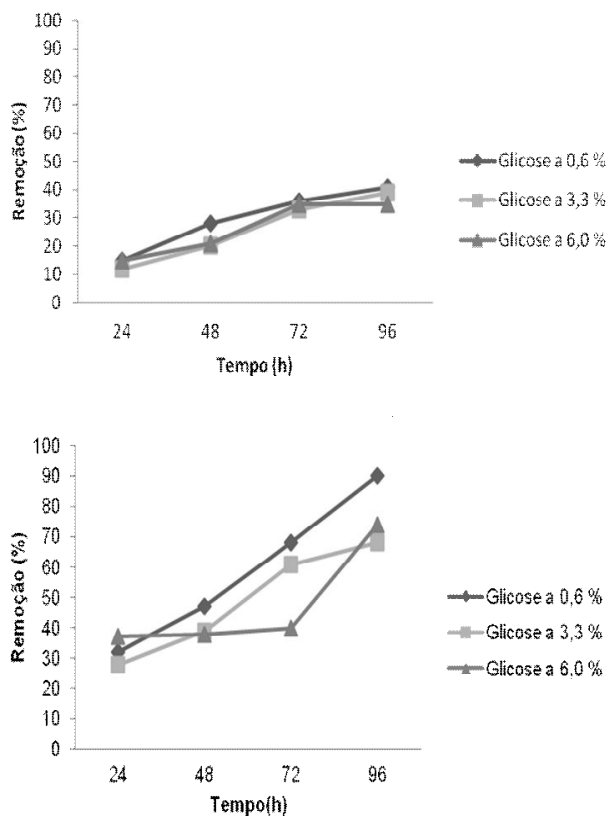

Figura 1: Cinética de remoção do fenantreno pela biomassa inativada de Cunninghamella elegans, utilizando soluções com diferentes concentrações de glicose (0,6; 3,3 e 6\%). [A] Fenantreno 0,1 mM $\mathrm{e}[\mathrm{B}]$ Fenantreno $0,2 \mathrm{mM}$.

Observou-se que a menor concentração de glicose $(0,6 \%)$ apresenta maior influência no processo de remoção de ambas as concentrações de fenantreno $(0,1$ e 0,2 mM). Destaca-se, ainda, que a biomassa inativada de $C$. elegans foi capaz de remover $90 \%$ de fenantreno na concentração a $0,2 \mathrm{mM}$ ao final do período de incubação. No entanto, as concentrações mais elevadas de glicose (3,3 e 6,0\%) sugerem um efeito repressor sob a remoção de fenantreno. Contudo, em todos os experimentos foi observada uma remoção gradual do fenantreno no decorrer de 96 horas.

A Figura 2 (A e B) apresenta os resultados obtidos com a biomassa viva de $C$. elegans frente a diferentes concentrações de fenantreno, na presença de 0,6 de glicose, demonstrando maior eficiência na remoção de fenantreno (82\%), com a maior concentração de glicose. Porém, a biomassa viva realizou uma biorremoção gradual do fenantreno na presença das concentrações de 3,3 e $6 \%$ de glicose. Contudo, a biomassa viva demonstra mais baixo desempenho na remoção de fenantreno, principalmente para a concentração $0,1 \mathrm{mM}$.
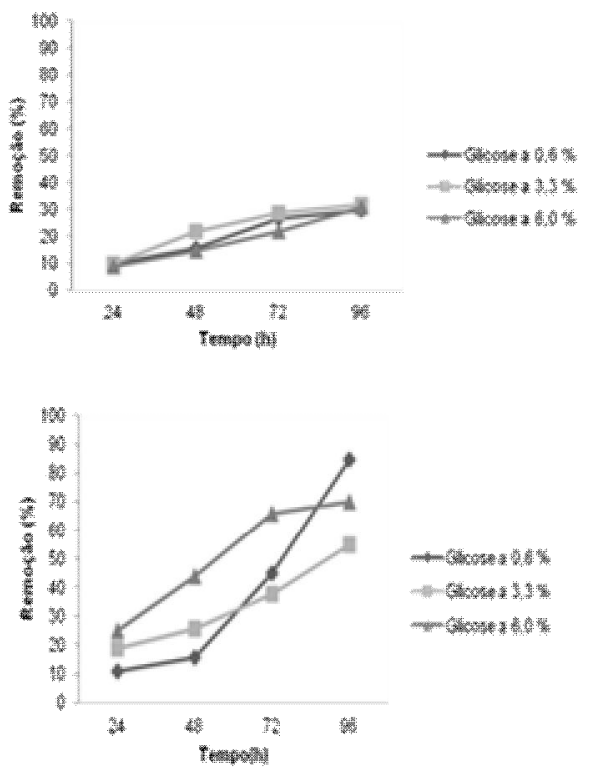

Figura 2: Cinética de remoção do fenantreno pela biomassa viva de Cunninghamella elegans, utilizando soluções com diferentes concentrações de glicose (0,6; 3,3 e 6\%). [A] Fenantreno 0,1 mM e [B] Fenantreno 0,2 mM. 
Nesse sentido, a ação da glicose na sua menor concentração, no processo de maior remoção do fenantreno pela biomassa inativada, provavelmente, ocorre devido ao maior número de sítios do biossorbente, possibilitando com maior vantagem de operação e de custo. Os dados obtidos com 0 alto desempenho da biomassa inativada de $C$. elegans são considerados relevantes à estabilidade e habilidade de adsorção, sendo essas características confirmadas pela literatura como importantes nos processos de biorremediação de sítios contaminados por HAPs (HWANG; CUTRIGHT, 2003; AMEZCUA-ALLIERI; LEAD; RODRÍGUEZVÁZQUEZ, 2005; GENTILI et al., 2006; LEITÃO, 2007; LEITAO, 2009).

Outra vantagem de fungos como biossorbente é a facilidade de separação da massa micelial por filtração, em comparação com leveduras e bactérias. Adicionalmente, os fungos filamentosos são menos sensíveis às variações de nutrientes, aeração, $\mathrm{pH}$, temperatura e têm um menor teor de ácidos nucléicos na biomassa. Além disso, resíduos hipersalinos são gerados em diversas atividades industriais, tais como fábricas de produtos químicos, petróleo e gás natural e as práticas de minimização de resíduos. Esses resíduos, vulgarmente designados como águas de produção, são constituídos por altas concentrações de sais, óleos, ácidos orgânicos, metais pesados e radionuclídeos (WOOLARD; IRVINE, 1995; LEITÃO; DUARTE; OLIVEIRA, 2007; LEITÃO, 2007; LEITAO, 2009).

Além disso, a capacidade de halotolerância é uma capacidade importante dos biossorbentes para corrigir os processos de poluição. Portanto, microorganismos halotolerantes apresentam propriedades fundamentais para o tratamento biológico sem, no entanto, causar prejuízos para o ecossistema (LEITÃO, 2007; LEITAO, 2009; LEITÃO, DUARTE; OLIVEIRA, 2007).

Neste caso, C. elegans UCP 542 é considerada um biossorbente extremamente importante, tendo em vista seu comportamento de halotolerância e considerando seu isolamento a partir de sedimentos de mangue do município de Rio Formoso, Pernambuco, Brasil (GOMES et al., 2000, FREITAS SILVA et al., 2007).

\subsection{EFEITOS DAS INTERAÇÕES ENTRE GLICOSE E BIOMASSA DE CUNNINGHAMELLA ELEGANSNO FENÔMENO DE REMOÇÃO DO FENANTRENO}

A Figura 3 e a Figura 4 apresentam os efeitos estatísticos entre as interações glicose e fenantreno, nas diferentes concentrações, tendo como variável resposta a porcentagem de remoção do fenantreno.

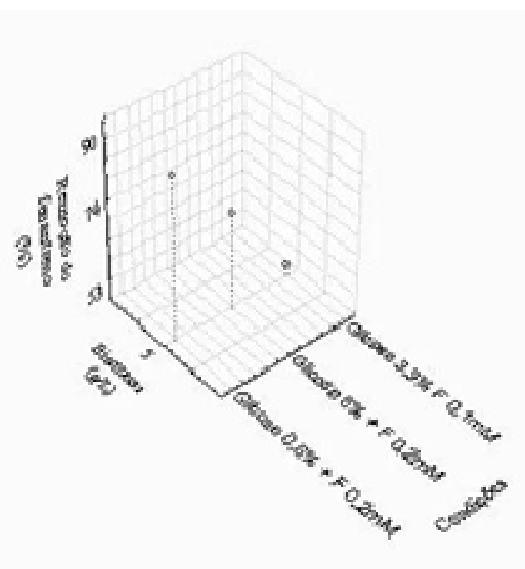

Figura 3. Interações entre a remoção de fenantreno utilizando biomassa inativada de Cunninghamella elegans, tendo a glicose 0,6 ; 3,3 e $6 \%$, como cosubstrato. 


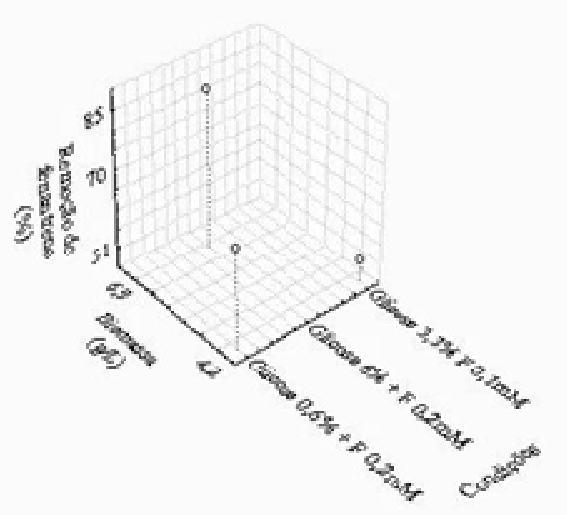

Figura 4. Interações entre a remoção de fenantreno utilizando biomassa viva de Cunninghamella elegans, tendo a glicose 0,$6 ; 3,3$ e $6 \%$, como co-substrato.

Os resultados obtidos indicaram que, entre todas as interações, a condição da biomassa inativada associada à concentração de $0,6 \%$ de glicose foi a mais eficiente. Contudo, os efeitos observados com a biomassa do fungo viva sugerem que ocorreu interação similar entre 0,6 e $6 \%$ de glicose. Trabalhos semelhantes demonstram que a fonte de carbono tem um papel importante na biorremoção de hidrocarbonetos (HERWIJNEN et al., 2003; CHAUDHARY et al., 2011).

\section{Considerações FinaIS}

A incorporação de glicose no processo de remoção de fenantreno pela biomassa de $C$. elegans investigada sugere que a linhagem apresenta elevado potencial na remoção do HAP. A maior eficiência foi com a menor concentração de glicose. A cinética de biorremoção mostrou-se linear em todos os experimentos, contudo, com o ponto central do planejamento utilizado, ocorreu um efeito negativo, com baixos níveis de remoção. A linhagem de C. elegans UCP (542) apresenta potencial para aplicação em processos de remediação de fenantreno e, provavelmente, para outros compostos aromáticos.

\section{AGRADECIMENTOS}

Os autores agradecem a concessão de bolsa ao PNPD-CAPES, FACEPE, o suporte financeiro ao CNPq e à CAPES, bem como, à infraestrutura da UNICAP.

\section{REFERÊNCIAS}

AMEZCUA-ALLIERI, M. A.; LEAD, J. R.; RODRÍGUEZ-VÁZQUEZ, R. Changes of chromium behavior in soil during phenanthrene removal by Penicillium frequentans. J. BioMetals., v. 18, p. 2329, 2005.

ANDRADE, V. S.; B. NETO, B.; SOUZA, W.; CAMPOS-TAKAKI, G. M. A factorial designs analysis of chitin production by Cunninghamella elegans. Can. J. Microbiol., v. 46, n. 11, p. 10421045, 2000.

BAMFORTH, S.; SINGLETON, I. Bioremediation of polycyclic aromatic hydrocarbons: current knowledge and future directions.J. Chem. Technol. Biotechnol., v. 80, n. 7, p. 723-736, 2005.
BARCLAY, C.D.; FARQUHAR, G.F.; LEGGE, R.L. Biodegradation and sorption of polyaromatic hydrocarbons by Phanerochaete chrysosporium. Applied Microbiology and Biotechnology, v. 42, p. 958-963, 1995.

BOONCHAN, S.; BRITZ, M. L.; STANLEY, G. A. Surfactant-enhanced biodegradation of high molecular weight polycyclic aromatic hydrocarbons by Stenotrophomonas maltophilia.Biotechnol Bioeng., v. 59, p.482-494. 1998.

BOONCHAN, S.; BRITZ, M.L.; STANLEY, G.A. Degradation and mineralization of high-molecularweight polycyclic aromatic hydrocarbons by 
defined fungal-bacterial cocultures.Appl. Environ. Microbiol., v. 66, p. 1007-1019, 2000.

BRODKORB, T. S.; LEGGE, R. L. Enhanced biodegradation of phenanthrene in oil tarcontaminated soils supplemented with Phanerochaete chrysosporium. Applied and Environmental Microbiology, v. 58, n .9, p. 31173121, 1992.

CAMPOS-TAKAKI, G. M.; DIETRICH, S. M.; BEAKES, G. W. Electron microscopic X-ray microprobe and cytochemical study of isolated cell walls of Mucoralean fungi. Mycol. Research, Inglaterra, v. 80, p. 536-541, 1983.

CERNIGLIA, C. E. Biodegradation of polycyclic aromatic hydrocarbons. Biodegradation, v. 3, p. 351-368, 1992.

CERNIGLIA, C. E. Fungal metabolism of polycyclic aromatic hydrocarbons: past, present and future applications in bioremediation.J. Ind. Microbiol. Biotechnol., v. 19, n. 5-6, p. 324-333, 1997.

CERNIGLIA, C. E.; GIBSON, D. T. Oxidation of benzo[a]pyrene by the filamentous fungus Cunninghamella elegans. J Biol Chem., v. 254, p. 12174-12180, 1979.

CERNIGLIA, C. E.; SUTHERLAND, J. B. Bioremediation of polycyclic aromatic hydrocarbons by ligninolytic fungi. In FUNGI IN BIOREMEDIATION, GADD, G.M. Ed.; Cambridge University Press: Cambridge, UK, p. 136-187, 2001.

CERNIGLIA, E.C.; VAN BAALEN, C.; GIBSON, T.D. Metabolism of naphthalene by the Cyanobacterium Oscillatoria sp. Strain. J. Gen. Microbiol., v. 116, p.485-494, 1980.

CHAUDHARY, P. et al.. Bioremediation of PAH by Streptomyces sp. Bulletin of Environmental Contamination and Toxicology,v. 86, n. 3, p.268271, 2011.

EL FANTROUSSI, S.; AGATHOS, S. N. Is bioaugmentation a feasible strategy for pollutant removal and site remediation? Curr Opin Microbiol, v. 8, p. 268-275, 2005.

FREITAS SILVA, M. C. et al. Effect of enviromental conditions on chitin and chitosan production by Cunninghamella elegans UCP542 Using Factorial Design. Asian Chitin J. v. 3, p. 15-22, 2007.

FREITAS SILVA, M. C. et al. Effect of phenanthrene on the germination, radial growth and chitin and chitosan production by
Cunninghamella elegans Lendner. In: MENDEZVILAS, A. (EDITOR) - 06002 Badajoz, Spain. (ORG.). Current Research Topics in Applied Microbiologyand MicrobialBiotechnology. World Scientific Publishing Company PTE LTD, Singapore, v. 1, p. 219-222, 2009.

GENTILI, A. R. et al. Bioremediation of crude oil polluted seawater by a hydrocarbon degrading bacterial strain immobilized on chitin and chitosan flakes. Int. Biodeter. Biodegr., v. 57, p. 222-228, 2006.

GOMES, P. F. et al. Aspectos da qualidade de ecossistemas do Município de Rio Formoso. International Conference- Sustainable use of estuaries and mangroves: challengens and propects. 2000. p. 82.

HERWIJNEN VAN, R. et al. Influence of phenanthrene and fluoranthene on the degradation of fluorene and glucose by Sphingomonas sp. strain LB126 in chemostat cultures. FEMS Microbiology Ecology, v. 46, p.105-111, 2003.

HESSELTINE, C. W.; ANDERSON, R. F. Microbiological production of carotenoids. Mycologia, v.49, p. 449-452,1957.

HOFRICHTER, M.; BUBLITZ, F.; FRITSCHE, W. Cometabolic degradation of o-cresol and 2,6dimethylphenol by Penicillium frequentans. J. Basic Microb., v. 35, p. 303-313, 1995.

HOLLIGER, C.; WOHLFAHRT, G.; DIEKERT, G. Reductive dechlorination in the energy metabolism of anaerobic bacteria. FEMS Microbiol Rev., v. 22, p. 383-398, 1998.

HWANG, S.; CUTRIGHT, T. J. Statistical implications of pyrene and phenanthrene sorptive phenomena: Effects of sorbent and solute properties. Archives of Environmental Contamination and Toxicology, v. 44, n. 2, p. 152159, 2003.

JOHNSEN, A.R.; WICK, L.Y.; HARMS, H. Principles of microbial PAH-degradation in soil.Environ. Pollution., v.133, n.1, p.71-84, 2005.

LEITÃO, A. L. Biodegradation of phenol and resorcinol by a halotolerant Penicillium. In:ENVIRONMENTAL BIODEGRADATION RESEARCH. New York, USA, Nova Science Publishers, p. 273-287, 2007.

LEITÃO, A. L. Potential of Penicillium Species in the Bioremediation Field.Int J Environ Res Public Health. v.6, n.4, p.1393-1417, 2009. 
LEITÃO, A. L.; DUARTE, M. P.; OLIVEIRA, J. S. Degradation of phenol by a halotolerant strain Penicillium chrysogenum.Int. Biodeter. Biodeg., v.59, p.220-225, 2007.

MALISZEWSKA-KORDYBACH, B.; SMRECZAK, B. Habitual function of agricultural soils as affected by heavy metals and polycyclic aromatic hydrocarbons contamination. Environ. Int., v.28, p.719-728, 2003.

MORAES FILHO, M. A. et al. Biotechnological potential of Phanerochaete chrysosporium UCP(Cunninghamella elegans UCP596 in the copper and zinc removal. Current Research Topics in Applied Microbiology and Microbial Biotechnology, Spain,v.1, p. 180-183, 2009.

PAZARLIOGLU, N.K.; TELEFONCU, A. Biodegradation of phenol by Pseudomonas putidaimmobilized on activated pumice particles.Process Biochem., v.40, p.1807-1814, 2005.

SUTHERLAND, J. B. Detoxification of polycyclic aromatic hydrocarbons by fungi.J. Ind. Microbiol., v.9, p.53-62, 1992.
TAO, X. et al. Rapid Degradation of Phenanthrene by Using Sphingomonas sp. GY2B Immobilized in Calcium Alginate Gel Beads.Int. J. Environ. Res. Public Health. v.6, p.2470-2480, 2009.

WILD, S.R.; JONES, K.C. Polynuclear aromatic hydrocarbons in the United Kingdom environment: a preliminary sourceinventory and budget. Environ. Pollut., v.88, p.91-108, 1995.

WOOLARD, C.R.; IRVINE, R.L. Treatment of hypersaline wastewater in the sequencing batch reactor.Water Res. v.29, p.1159-1168, 1995.

WU, Z.; WANG, Y.; XING, Z. Study on degradingphenol by immobilized Ralstoniametallidurans $\mathrm{CH} 34$.Microbiology, v.32, p.31-36, 2005.ZHANG, S.Y.; WANG, Q.F.; XIE, S. G. Microbial community changes in contaminated soils in response to phenanthrene amendment.Int. J. Environ. Sci. Technol., v.8, n. 2, p. 321-330, 2011.

ZHU, H.; AITKEN, M.D. Surfactant-enhanced desorption and biodegradation of polycyclic aromatic hydrocarbons in contaminated soil.Environ Sci Technol., v.44, n. 19, p.7260-7265, 2010. 